\title{
Reptational dynamics in dissipative particle dynamics simulations of polymer melts
}

\author{
Petri Nikunen \\ Biophysics and Statistical Mechanics Group, Laboratory of Computational Engineering, \\ Helsinki University of Technology, P.O. Box 9203, FI-02015 HUT, Finland \\ Ilpo Vattulainen \\ Laboratory of Physics and Helsinki Institute of Physics, Helsinki University of Technology, P.O. Box 1100, \\ FI-02015 HUT, Finland; MEMPHYS-Center for Biomembrane Physics, University of Southern Denmark, \\ Odense, Denmark; Institute of Physics, Tampere University of Technology, P.O. Box 692, FI-33101 Tampere, Finland \\ Mikko Karttunen \\ Biophysics and Statistical Mechanics Group, Laboratory of Computational Engineering, \\ Helsinki University of Technology, P.O. Box 9203, FI-02015 HUT, Finland; \\ Department of Applied Mathematics, University of Western Ontario, London (ON), Canada
}

(Dated: November 27, 2005)

\begin{abstract}
Understanding the complex viscoelastic properties of polymeric liquids remains a challenge in materials science and soft matter physics. Here, we present a simple and computationally efficient criterion for the topological constraints in polymeric liquids using the Dissipative Particle Dynamics (DPD). The same approach is also applicable in other soft potential models. For short chains the model correctly reproduces Rouse-like dynamics whereas for longer chains the dynamics becomes reptational as the chain length is increased-something that is not attainable using standard DPD or other coarse-grained soft potential methods. Importantly, no new length scales or forces need to be added.
\end{abstract}

\section{INTRODUCTION}

The static and dynamic properties of polymeric liquids are, by and large, dominated by topological constraints. The origin of these constraints is easy to understand: polymers can slide past but not penetrate through each other. That is the physical origin of the reptation model [1, 2, 3] which is the most successful theory in describing the behavior of entangled polymers. Despite active research in the field, entangled polymeric liquids keep posing many challenges to theorists [4, 5, 6], experimentalists 17, 8, 9] and computational modelers $[10,11,12,13,14,15,16]$. The importance of understanding the fundamentals of polymeric liquids can hardly be overemphasized as they are one of the key issues in novel (bio)materials science [17, 18].

The dynamics of polymer melts is typically described in terms of the Rouse and reptation models [3]. Short chains are able to move to any direction and are not entangled. That is the physical origin of the Rouse model [3, 19]. For longer chains, entanglements and uncrossability of chains cannot be ignored, and the chains become constrained to move in the direction of the chain backbone. This motion resembles that of a reptating snake-hence the name reptation model [1, 2, 3].

Computer simulations offer a detailed look into polymers and their dynamics. In classical molecular dynamics simulations the system size and simulation time pose limits as they are typically of the order of $10 \mathrm{~nm}$ in linear size and around $10 \mathrm{~ns}$ in time. In contrast, coarse grained methods, such as dissipative particle dynamics (DPD), allow access to micrometer and microsecond scales. That is due to the soft potentials, and, like everything in life, they do not come without a price to pay: the softness of the conservative potentials allows the chains to slide through each other thus strongly affecting the dynamics of the system. Indeed, the scaling laws obtained from DPD simulations of polymer melts [20, 21] are not able to describe entangled liquids. This is a direct consequence of the fact that in DPD simulations, polymers can penetrate through themselves. Whereas that is not a problem in studying the equilibrium properties in the Rouse regime, reptation cannot be studied using the basic DPD model with soft interactions.

To preserve the advantages of coarse-grained models and to correct for their deficiencies, Padding and Briels [13] recently introduced an algorithm that explicitly detects and prevents bond crossings. They consider bonds as elastic bands that become entangled and use energy minimization to determine the entanglement positions. This approach is general and very promising but it is also complicated to implement and computationally intensive [13].

Another promising approach was put forward by Pan and Manke [22]. They reduce the frequency of bond crossings by introducing segmental repulsive forces between the points of nearest contact between neighboring chains. This approach is simple to implement but the introduction of a new force and a related cutoff increases the computational load, and adds a new length scale whose physical determination is somewhat ambiguous. On the other hand, the model seems to be able to capture both the Rouse and reptational behavior [22] like that of Padding and Briels [13, 23].

In this article, we introduce a simple and generic criterion based on simple geometrical arguments to solve the crossability problem. No new forces are added, the approach is conceptually simple and does not depend on the level of coarse graining. Importantly, it allows easy, and if necessary even on-the-fly, tuning between the Rouse and reptation regimes.

The rest of this paper is organized as follows. In the next section we will briefly describe the DPD method. SectionIII describes our criterion for including topological constraints in 
DPD, or for that matter any other soft potential, simulation. In Sec. $\nabla$ we show results from our simulations and compare them to other methods. Finally, we finish with a discussion and outlook in Sec.[V]

\section{DISSIPATIVE PARTICLE DYNAMICS}

In DPD, the time evolution of particles is given by the Newton's equations of motion, and the total force acting on particle $i$ is given as a sum of pairwise conservative, dissipative, and random forces, respectively, as $\vec{F}_{i}=\sum_{i \neq j}\left(\vec{F}_{i j}^{C}+\vec{F}_{i j}^{D}+\vec{F}_{i j}^{R}\right)$.

The conservative force is independent of the dissipative and random forces. Typically it takes the form

$$
\vec{F}_{i j}^{C}= \begin{cases}a_{i j}\left(1-r_{i j} / r_{c}\right) \vec{e}_{i j}, & \text { if } r_{i j}<r_{c} \\ 0, & \text { otherwise }\end{cases}
$$

with $\vec{r}_{i j} \equiv \vec{r}_{i}-\vec{r}_{j}, r_{i j} \equiv\left|\vec{r}_{i j}\right|$, and $\vec{e}_{i j} \equiv \vec{r}_{i j} / r_{i j}$. The variable $a_{i j}$ describes the repulsion between particles $i$ and $j$, and thus produces excluded volume interactions.

The dissipative force is expressed as

$$
\vec{F}_{i j}^{D}=-\gamma \omega^{D}\left(r_{i j}\right)\left(\vec{v}_{i j} \cdot \vec{e}_{i j}\right) \vec{e}_{i j}
$$

where $\gamma$ is a friction parameter, $\omega^{D}\left(r_{i j}\right)$ a weight function for the dissipative force, and $\vec{v}_{i j} \equiv \vec{v}_{i}-\vec{v}_{j}$. The dissipative force slows down the particles by decreasing kinetic energy from them. This effect is balanced by the random force due to thermal fluctuations,

$$
\vec{F}_{i j}^{R}=\sigma \omega^{R}\left(r_{i j}\right) \zeta_{i j} \vec{e}_{i j},
$$

where $\sigma$ is the amplitude of thermal noise, $\omega^{R}\left(r_{i j}\right)$ is the weight function for the random force, and $\zeta_{i j}(t)$ are Gaussian random variables with $\left\langle\zeta_{i j}(t)\right\rangle=0$ and $\left\langle\zeta_{i j}(t) \zeta_{k l}\left(t^{\prime}\right)\right\rangle=$ $\left(\delta_{i k} \delta_{j l}+\delta_{i l} \delta_{j k}\right) \delta\left(t-t^{\prime}\right)$. The condition $\zeta_{i j}(t)=\zeta_{j i}(t)$ is required for momentum conservation. That is a necessary condition for the conservation of hydrodynamics.

The weight functions $\omega^{D}\left(r_{i j}\right)$ and $\omega^{R}\left(r_{i j}\right)$ cannot be chosen arbitrarily. Español and Warren [24] showed that the fluctuation-dissipation relations $\omega^{D}\left(r_{i j}\right)=\left[\omega^{R}\left(r_{i j}\right)\right]^{2}$ and $\sigma^{2}=2 \gamma k_{B} T$ must be satisfied for the system to have a canonical equilibrium distribution. Here $T$ is the temperature of the system and $k_{B}$ is the Boltzmann constant. The functional form of the weight functions is not defined by the DPD method but virtually all DPD studies use

$$
\omega^{D}\left(r_{i j}\right)=\left[\omega^{R}\left(r_{i j}\right)\right]^{2}= \begin{cases}\left(1-r_{i j} / r_{c}\right)^{2}, & \text { if } r_{i j}<r_{c} \\ 0, & \text { otherwise. }\end{cases}
$$

Coarse graining in DPD comes in through the soft conservative potential and forces (Eq. (1)). A detailed account of DPD, derivation of time and length scales, and its applications is given by R.D. Groot [25]. An in depth discussion of coarse graining by P. Español can be found in the same reference.

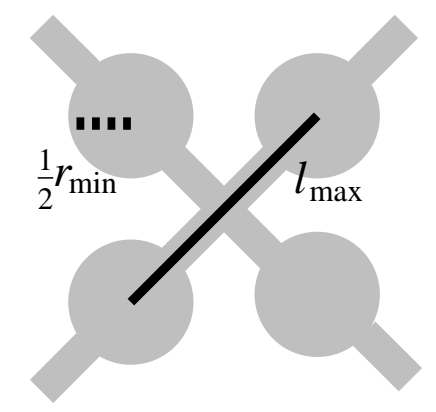

FIG. 1: Two bonds crossing each other. If Eq. 5] is satisfied, crossings cannot occur.

\section{TOPOLOGICAL CONSTRAINTS}

To take into account the topological constraints, chain crossings must be prevented. As discussed in the introduction, there are currently two off-lattice methods [13, 22] for this purpose. Here, we introduce a third alternative.

First, each individual bead has a radius $r_{\min }$ which is impenetrable to other beads. In systems with Lennard-Jones potentials that condition is automatically satisfied due to the $r^{-12}$ part that takes care of the Fermi exclusion principle. In mesoscopic simulations with soft potentials that constraint needs special attention. Second, the intramolecular bonds have some maximum stretch, $\ell_{\max }$. By using simple geometry, we can postulate that if the condition

$$
\sqrt{2} r_{\min }>\ell_{\max }
$$

is satisfied, any two bonds cannot cross each other, see Fig. 1 The length scales involved, i. e., $r_{\min }$ and $\ell_{\max }$ have a clear physical meaning.

The obvious question is whether that condition is actually useful and when does it work. As an example, let us consider DPD simulations of polymers. The parameters used in these simulations are often justified on the basis of the FloryHuggins theory [25, 26], where the key component is the solubility as expressed by the $\chi$-parameters. Then, in simulations of block co-polymers, e.g., it is the mutual repulsion between the different components that matters-as a matter of fact, the values of the interaction parameters $a_{i j}$ may be derived in different ways and their values tell only about the degree of coarse-graining. The condition set by Eq. (5) can thus be met by a proper degree of coarse graining, complemented by a reasonable description for bond stretching (springs). Indeed, above $\ell_{\max }$ is limited by the type of springs used in the model. With FENE springs [27] that is easy to tune as they have only finite extension after which the force becomes infinite. With harmonic springs more care is needed to satisfy Eq. (5) as there is no FENE-like cutoff set by the equation of motion. We will return to that in the Sec. $\nabla$ 


\begin{tabular}{|l|l|l|l|l|}
\hline \hline$a$ (amplitude of the conservative force) & 50 & 100 & 150 & 200 \\
$k$ (spring constant) & 100 & 200 & 300 & 400 \\
$\Delta t$ (time step) & 0.03 & 0.02 & 0.015 & 0.0125 \\
\hline \hline
\end{tabular}

TABLE I: The parameters used in this study.

\section{SIMULATIONS}

For simplicity, and to be able to compare the model with other simulations, we considered a melt of linear polymers in a cubic box (3D) with periodic boundary conditions. To avoid finite size effects, the linear box size $L$ was chosen to be at least 1.75 times the average end-to-end distance of chains. We also carried out simulations with different box sizes to ensure that the systems were free of finite size effects. That was done since it is known that static properties are affected by them [28].

All the systems had 128 chains consisting of $N$ monomers, and no additional solvent or free monomers were present. All monomers were chosen to be identical, and thus the monomer mass was set equal to unity, $m=1$, fixing the scale of mass. The cutoff distance $r_{c}$ sets the length scale for the model. The conservative forces had the form given in Eq. (1), with $r_{c}=1$ and $a_{i j}=a$ for all particle pairs. The values of $a$, as well as other simulation parameters used in these simulations are listed in Table IV

For the random and dissipative forces we used Eqs. (2) and (3) with the common choices [25, 26, 29, 30] $\gamma=4.5$ and $\sigma=3$. This sets the temperature to $k_{B} T=1$, and hence the time scale is given by $\sqrt{m r_{c}^{2} / k_{B} T}$.

The monomers were connected using harmonic springs, i. e., $\vec{F}_{i}^{S}=\sum_{j} k\left(\ell-r_{i j}\right) \vec{e}_{i j}$, where the sum runs over all particles $j$ to which particle $i$ is connected. The equilibrium bond length was set to $\ell=0.95$. That particular value was chosen as it is very near the first maximum of the radial distribution function (at the density $\rho=1$ ). The spring constant was chosen to be $k=2 a$. If $k$ is much smaller, bonds are very flexible and Eq. (5) is not satisfied. On the other hand, if $k$ is much larger than $a$, the time step $\Delta t$ must be decreased from the value set by the choice of $a$ thus decreasing the computational efficiency. Another possibility would be to use FENE springs [28] since they have finite extension.

The density was chosen to be $\rho=1$, which is lower than the densities typically used in DPD simulations [25, 26]. The reason for high densities is to give different repulsive interactions for different particle types. This works only if particles overlap each other considerably. In the present work, we don't need such interactions, and therefore the lower density is sufficient. In fact, the density of $\rho=1$ sets the monomer-monomer coordination number near 12 , which is a typical value for real liquids.

All systems were started from random flight initial configurations and they were equilibrated for $10^{6}$ time steps. After the equilibration, we simulated systems at least for $10^{7}$ time steps to compute the desired quantities. Equations of motion were integrated using the DPD-VV algorithm [29, 31, 32].

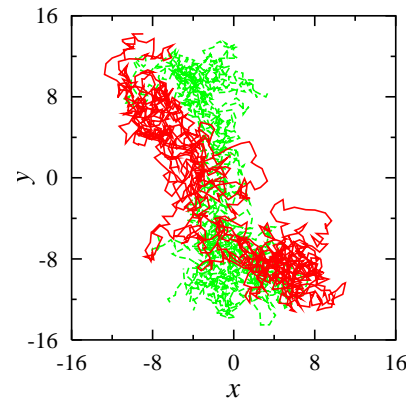

a)

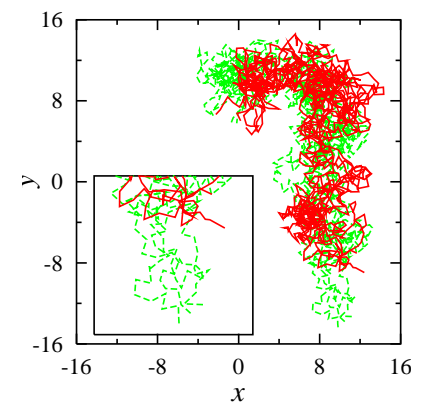

b)
FIG. 2: The snapshots present 10 superpositions of configurations for a chain of length $N=256$ taken at times 100 apart. Green: times up to 500 (in DPD time units), red: times from 500 to 1000 . a) Rouse dynamics $(a=25, k=50)$ and $\mathbf{b})$ reptation $(a=100, k=200)$.

\section{RESULTS}

Figure 2 shows snapshots of the chain motion during the simulation at different times and regimes. For clarity, the chain is projected onto two dimensions. It is immediately clear that the motions in Figs. 2a and 2b are qualitatively different. Figure 2a shows Rouse-like motion in which the polymers are free to move in every direction, and Fig. 2 b represents reptation confined into a tube.

\section{A. Radial distribution function}

We will now study the static properties to see the effect of Eq. (5). As discussed, by tuning the chain stiffness it is possible to move gradually from the Rouse regime to reptation. This should be reflected in both the radial distribution function and the bond length distribution.

The radial distribution function (RDF) $g(r)$ describes the qualitative structure of a fluid. It is defined as $g(r)=\rho(r) / \rho$ where $\rho(r)$ is the average density from a given particle at a distance $r$. Figures 3 and 3 b show the radial distribution function $g(r)$ and the bond length distribution $f_{\ell}(r)$ for different parameter sets for chains of length $N=32$. The arrows in the figures indicate the values of $r_{\min }$ and $\ell_{\max }$ in Eq. (5). As the figures show, Eq. (5) is satisfied for larger values of $a$ and $k$. The small non-zero values below $r_{\min }$ are due to the softness of the interparticle DPD potentials.

The above can be characterized by taking a look at the average bond lengths are their mean square deviations. For $a_{i j}=50$ we measured $\langle\ell\rangle=0.977 \pm 0.092$. As the strength of interaction is increased we obtain $\langle\ell\rangle=0.968 \pm 0.064$ for $a_{i j}=100,\langle\ell\rangle=0.966 \pm 0.051$ for $a_{i j}=150$, and $\langle\ell\rangle=0.965 \pm 0.045$ for $a_{i j}=200$. The most important issue is the decrease of the mean square deviation as that restricts the amount of overlap between the monomers of different chains. Importantly, for FENE chains this can be directly controlled by using the above measurements and RDF as a guideline and setting the maximum extent of the chain to an appropriate value. 


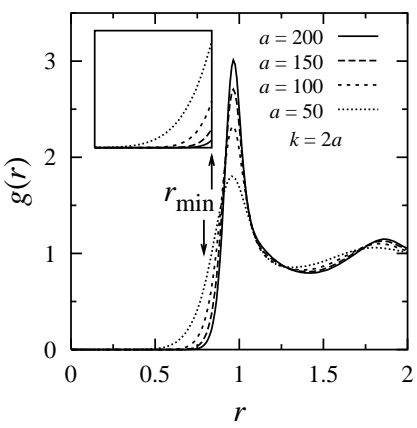

a)

FIG. 3: a) Radial distribution function in the case of $N=32$. The arrow shows the distance $r_{\min }$, and the inset the region at lengths shorter than $r_{\min }$. Compare with LJ models $\left(r_{c}=1,2.5\right)$. b) Bond length distribution $(N=32)$. The arrow shows the location of $\ell_{\max }$, and the inset the region at values larger than $\ell_{\max }$.

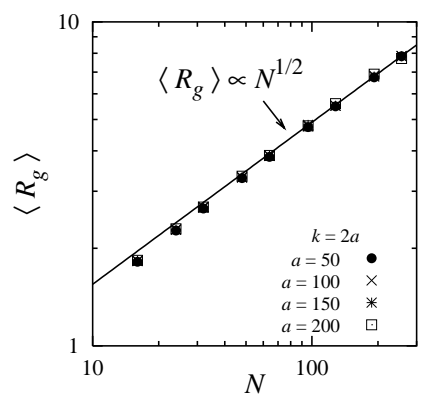

a)

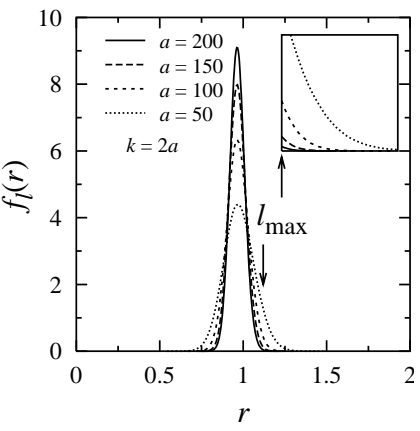

b)

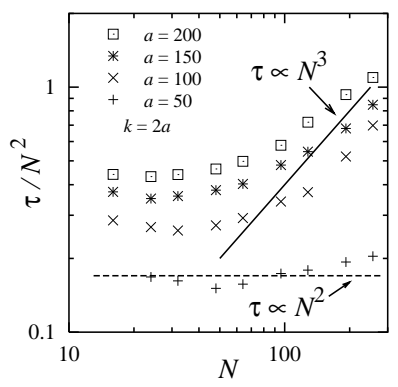

a)

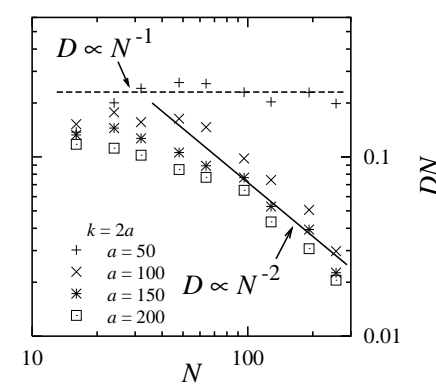

b)
FIG. 5: a) Scaling of the longest relaxation time $\tau$. There is a crossover from Rouse scaling $\left(\tau \propto N^{2}\right)$ to reptation $\left(\tau \propto N^{3}\right)$. b) Similarly, the proper scaling limits are reached for the diffusion coefficient $D$.

\section{Relaxation time}

One of the main practical obstacles in simulations of polymeric solutions is the long stress relaxation time. The longest relaxation time, $\tau$, depends on the molecular weight and the reptation theory predicts it to scale as $\tau \propto N^{3}$. That prediction assumes only one mechanism for relaxation, i. e., diffusion along the countour [1]. The Rouse model predicts a distinctly different behavior with $\tau \propto N^{2}$.

To estimate the scaling behavior, we measured the end-toend autocorrelation function. It is shown in Fig. 4 $\mathrm{b}$ for polymers of different length. Assuming exponential decay, i. e.,

$$
\langle\vec{R}(t) \cdot \vec{R}(0)\rangle \sim \exp (-t / \tau)
$$

different parameters. b) The end-to-end vector autocorrelation ( $a=$ $100, k=200$ ) for chains of different length.

A comparison of the radial distribution functions shows that the current approach allows for tuning between typical DPD results [21, 26, 32] and typical molecular dynamics simulations using Lennard-Jones potentials [28]. As the bond strength is increased ( $a \geq 100, k \geq 200), g(r)$ becomes qualitatively similar to that from a Lennard-Jones system.

\section{B. Static scaling}

Next, we studied the end-to-end distance $R$ and the radius of gyration $R_{g}$. The former is defined as $R=|\vec{R}|=\left|\overrightarrow{r_{1}}-\overrightarrow{r_{N}}\right|$ and the latter as $R_{g}^{2}=\frac{1}{N} \sum_{i=1}^{N}\left(\vec{r}_{i}-\vec{r}_{\mathrm{cm}}\right)^{2}$, where $\vec{r}_{\mathrm{cm}}=$ $\frac{1}{N} \sum_{i=1}^{N} \vec{r}_{i}$. In a polymer melt, they are expected to scale as $\langle R\rangle \propto N^{1 / 2}$ and $\left\langle R_{g}\right\rangle \propto N^{1 / 2}$ in both Rouse and the reptation regime. Previous studies using soft potentials [20] and systems with more realistic hard potentials [28] exhibit scaling. In Fig. 4 4 we plot the results for the radius of gyration for different parameter sets. It is clear from the figure that the system exhibits the proper scaling behavior independently of the interaction parameters as it should. we can extract the longest relaxation time $\tau$ by fitting. Figure 5 a shows that both scaling regimes are captured properly. Fig. 51 illustrates one of the main results of this paper: the simple criterion summarized by Eq. (5) allows an easy, physical and computationally efficient tuning between the Rouse regime and reptation.

The scaling exponents 2 and 3 for Rouse and reptation, in respective order, are the limiting laws. The exponents have been frequently debated in the literature. For example, for the same values of $N$ as used here, Padding and Briels [23] found two scaling regimes in $\tau$, one with exponent 2.8 and the other one with exponent 3.5. The dependence $\tau \propto N^{3.4}$ is experimentally observed for the longest relaxation time in the entangled regime [33]. This discrepancy is often associated with fluctuations of the contour length of the primitive path. In a real situation, however, the tube has a characteristic lifetime and the length of the primitive path fluctuates since the Rouse modes continue in the direction along the primitive path.

Determining the value of the exponent was not the main goal here, and thus we did not attempt to evaluate it in a high precision-we will focus on that in a future publication. The above simply to demonstrates that it is indeed possible to use the soft DPD model to describe entangled polymer liquids realistically without introducing additional length scales and forces. 


\section{Diffusion}

The motion of a polymer, or its segments, is described by the diffusion coefficient. Typically, one measures the centerof-mass diffusion coefficient for a polymer chain, i. e.,

$$
D=\lim _{t \rightarrow \infty} \frac{1}{6}\left\langle\left[\vec{r}_{\mathrm{cm}}(t)-\vec{r}_{\mathrm{cm}}(0)\right]^{2}\right\rangle .
$$

The scaling of $D$ with molecular weight has been studied intensely over the years, see e.g. the discussion in [4].

The theory predicts two scaling limits, $D \propto N^{-1}$ for the Rouse model and $D \propto N^{-2}$ for the pure reptation model ( $N$ is proportional to molecular weight). Figure $5 \mathrm{~b}$ shows that both scaling regimes are found.

Considering the nature of the DPD model, it is remarkable that both regimes are recovered. In simulations using the plain DPD model without paying attention to the criterion given by Eq. [5, only Rouse scaling has been observed [20, 21] even in the case of long polymers.

As with the longest relaxation time, the exponents typically reported are between the scaling limits. Pearson et al. [34] measured $D$ as a function of molecular weight $M_{w}$ in polyethylene and they found that the diffusion coefficient follows a power law $D=1.65 M_{w}^{-1.98} \mathrm{~cm}^{2} / \mathrm{s}$ for the entire range from $M_{w}=600$ to $M_{w}=12000(\mathrm{~g} / \mathrm{mol})$. The simulations by Kremer et al. [11, 28] and Padding and Briels [13, 23] confirmed this finding: the center of mass diffusion coefficient scales as $D \propto N^{-2}$ in melt.

Padding and Briels [23] compared their results to different simulations and experiments, and found that in ethylene the crossover between Rouse-like and reptational dynamics takes place at molecular weight of $560 \mathrm{~g} / \mathrm{mol}$ (corresponds to 40 ethylenes). Because in Fig. 5b the crossover takes place between $N=40$ and $N=60$, we can picture each particle roughly as one ethylene unit.

\section{DISCUSSION}

In this article we have presented a simple criterion for topological constraints in coarse grained DPD simulations of poly- meric liquids. No new forces or length scales were added. We showed that this approach is able to reproduce the Rouse model at one limit and reptational dynamics at the other. Here, we validated and demonstrated this approach against other models and experimental results using linear homopolymers. This approach can also be used for systems of, e. g., block copolymers with different interactions and monomer sizes, and shear simulations. In practice, one can always run a short test simulation, and use $g(r)$ and the bond length distribution (as in Figs. 3a and b) to verify that the criterion set by Eq. (5) is met.

There is one other issue that we need to address, namely by tuning the chain stiffness one inevitably changes the entanglement length in addition to intercrossability of chains. It is known from previous simulations using Lennard-Jones as well as some coarse-grained models that increasing chain stiffness intensifies reptation [35, 36, 37]. To account for this in order to use coarse-grained methods such as DPD with soft potentials in a controlled way, one should use (at least) the persistence length as measure that should be matched between the coarse-grained and the atomistic models. Here, we did not attempt to do that systematically.

Here, we made no attempt to determine the precise scaling exponents for the diffusion coefficient or the longest relaxation time. Though, there are a lot of subtleties, such as the tube dimensions, lifetime, friction and the plateau modules, related to the scaling behavior [11]. A future publication will focus on them and the detailed mechanisms.

\section{Acknowledgments}

This work has been supported by Emil Aaltonen foundation (M. K.), the Academy of Finland through its Center of Excellence Program (I.V.) and the Academy of Finland Grants Nos. 54113, 00119 (M.K.), and 80246 (I.V.). We also thank the Finnish IT Center for Science (CSC) and the HorseShoe (DCSC) supercluster computing facility at the University of Southern Denmark for computer resources.
[1] S. F. Edwards, Proc. Phys. Soc. 92, 9 (1967).

[2] P. G. de Gennes, J. Chem. Phys. 55, 572 (1971).

[3] M. Doi and S. F. Edwards, The Theory of Polymer Dynamics (Clarendon Press, Oxford, 1986).

[4] T. C. B. McLeish, Adv. Phys. 51, 1379 (2002),

[5] R. Everaers, S. K. Sukumaran, G. S. Grest, C. Svaneborg, A. Sivasubramanian, and K. Kremer, Science 303, 823 (2004),

[6] S. K. Sukumaran, G. S. Grest, K. Kremer, and R. Everaers, J. Polym. Sci. B 43, 917 (2005).

[7] D. Richter, B. Farago, L. J. Fetters, J. S. Huang, B. Ewen, and C. Lartigue, Phys. Rev. Lett. 64, 1389 (1990),

[8] J. Käs, H. Strey, and E. Sackmann, Nature 368, 226 (1994),

[9] P. Schleger, B. Farago, C. Lartigue, A. Kollmar, and D. Richter,
Phys. Rev. Lett. 81, 124 (1998),

[10] K. Kremer, G. S. Grest, and I. Carmesin, Phys. Rev. Lett. 61, 566 (1988),

[11] M. Pütz, K. Kremer, and G. S. Grest, Europhys. Lett. 49, 735 (2000),

[12] W. Paul, K. Binder, D. W. Heermann, and K. Kremer, J. Chem. Phys. 95, 7726 (1991).

[13] J. T. Padding and W. J. Briels, J. Chem. Phys. 115, 2846 (2001),

[14] W. Paul and G. D. Smith, Rep. Prog. Phys. 67, 1117 (2004),

[15] H. C. Öttinger, J. Non-Newtonian Fluid Mech. 120, 207 (2004).

[16] K. Kremer, S. K. Sukumaran, R. Everaers, and G. S. Grest, Computer Physics Comm. 169, 75 (2005).

[17] S. Förster and T. Plantenberg, Angew. Chem. Int. Edn. 41, 689 
(2002).

[18] S. Rastogi, D. R. Lippits, G. W. M. Peters, R. Graf, Y. Yao, and H. W. Spiess, Nature Materials 4, 635 (2005).

[19] P. E. Rouse, J. Chem. Phys. 21, 1272 (1953).

[20] N. A. Spenley, Europhys. Lett. 49, 534 (2000),

[21] X. Guerrault, B. Rousseau, and J. Farago, J. Chem. Phys. 121, 6538 (2004),

[22] G. Pan and C. W. Manke, Int. J. Mod. Phys. B 17, 231 (2003),

[23] J. T. Padding and W. J. Briels, J. Chem. Phys. 117, 925 (2002).

[24] P. Español and P. Warren, Europhys. Lett 30, 191 (1995),

[25] R. D. Groot, in Novel Methods in Soft Matter Simulations, edited by M. Karttunen, I. Vattulainen, and A. Lukkarinen (Springer Verlag, Heidelberg, 2004), Lecture Notes in Physics, pp. 5-38.

[26] R. D. Groot and P. B. Warren, J. Chem. Phys. 107, 4423 (1997),

[27] G. S. Grest and K. Kremer, Phys. Rev. A 33, 3628 (1986),

[28] K. Kremer and G. S. Grest, J. Chem. Phys. 92, 5057 (1990).
[29] G. Besold, I. Vattulainen, M. Karttunen, and J. M. Polson, Phys. Rev. E 62, R7611 (2000),

[30] J. C. Shillcock and R. Lipowsky, Nature Materials 4, 225 (2005),

[31] I. Vattulainen, M. Karttunen, G. Besold, and J. M. Polson, J. Chem. Phys. 116, 3967 (2002),

[32] P. Nikunen, M. Karttunen, and I. Vattulainen, Computer Phys. Comm. 153, 407 (2003),

[33] M. Doi, J. Polym. Sci. 19, 265 (1981).

[34] D. S. Pearson, G. Ver Strate, E. von Meerwall, and F. C. Schilling, Macromolecules 20, 1133 (1987).

[35] R. Faller, F. Müller-Plathe, and A. Heuer, Macromolecules 33, 6602 (2000),

[36] R. Faller and F. Müller-Plathe, ChemPhysChem 2, 180 (2001),

[37] R. Faller and F. Müller-Plathe, Polymer 43, 621 (2002). 